\title{
COVID-19 Mortality in Pediatrics
}

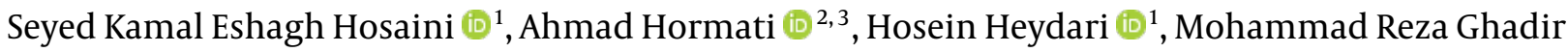 (iD) ${ }^{3}$, Mahboubeh Afifian (iD ${ }^{4}$ and Sajjad Ahmadpour (iD) ${ }^{3, *}$}

\author{
${ }^{1}$ Department of Pediatrics, School of Medicine, Hazrat-e Fateme Masoume Hospital, Qom University of Medical Sciences, Qom, Iran \\ ${ }^{2}$ Gastrointestinal and Liver Diseases Research Center, Iran University of Medical Sciences, Tehran, Iran \\ ${ }^{3}$ Gastroenterology and Hepatology Diseases Research Center, Qom University of Medical Sciences, Qom, Iran \\ ${ }^{4}$ Health Information Technology, Tehran University of Medical Sciences, Tehran, Iran \\ "Corresponding author: Gastroenterology and Hepatology Diseases Research Center, Qom University of Medical Sciences, Qom, Iran. Email: sajjadahmadpour@yahoo.com
}

Received 2020 April 29; Revised 2020 December 03; Accepted 2021 January 18.

\begin{abstract}
Introduction: New Coronavirus disease 2019 (COVID-19) has caused serious health problems worldwide. The disease first started in Wuhan, China, and then resulted in thousands of deaths throughout the world.

Case Presentation: According to data from involved patients, children and neonates include a small proportion of patients with COVID-19. However, in some cases, hospitalization and even death still may occur. In this case report, we described mortality associated with COVID-19 in two pediatrics from Qom city, Iran. One of them presented with severe symptoms that needed high-flow ventilation and admission to the ICU.

Conclusions: The findings of this report confirm that COVID-19 may cause death in pediatrics, like in adults. Timely diagnosis, precise evaluation of clinical symptoms, and effective treatment of COVID-19-infected children can play an important role in reducing mortality rates.
\end{abstract}

Keywords: COVID-19, Pediatric, Intensive Care Unit, Mortality

\section{Introduction}

Coronavirus disease 2019 (COVID-19), as a new disease, has affected thousands of people worldwide. The disease first started in Wuhan, Hubei Province, China, on 31 December 2019 and then afflicted humans throughout the world (1). The first confirmed case of acute respiratory syndrome due to COVID-19 was reported from Qom Province, Iran, on 22 February 2020. Since then, a lot of efforts have been made to control and manage the diseases, particularly by the widespread announcement. From 22 February to 17 August, 2020, the COVID-19 epidemic has resulted in $>$ 345,000 cases and $>19,000$ deaths in Iran.

Data from Iran suggest that infants, children, and adolescents aged $<18$ years showed a low number of COVID19 cases $(2,3)$. Fever, cough, shortness of breath, and diarrhea are the commonly reported symptoms among confirmed COVID-19 adults, while these signs and symptoms are less frequently reported among infected pediatrics than in adults $(4,5)$. Studies have shown that children and neonates comprise a small proportion of cases among patients with COVID-19; however, in some cases, hospitalization still may occur. Timely diagnosis, precise evaluation of clinical symptoms, and effective treatment of COVID-19 infected children can play an important role in reducing mortality rates and breaking the transmission chain in the community. It is necessary to mention that, like in adults, social distancing is an important factor for the management of the disease in the pediatric population $(6,7)$.

In this scientific letter, we describe mortality associated with COVID-19 in two pediatrics from Iran who were referred to Hazrat-e Fatemeh Masoumeh Hospital, the Qom city. Following history-taking, physical examinations and biochemical tests were conducted. For confirmation of COVID-19 infection, a chest CT scan and laboratory tests for SARS-CoV-2 were performed using real-time reverse-transcriptase polymerase chain reaction (rRT-PCR). Also, clinical features, including symptoms, hospitalizations, and medications, were recorded for the patients.

\section{Case Presentation}

On 22 March 2020, an eight-year-old female was referred to Shahid Beheshti hospital, Qom city, with weak- 
ness, lethargy, abdominal pain, nausea, and fever (sublingual temperature more than $38^{\circ} \mathrm{C}$ ). She had had these symptoms for two days (from 19 March 2020). She did not have any medical history, specific risk factors, or background of receiving any drugs.

Upon admission to the hospital, she looked ill, and her vital signs were as follows: Blood pressure $85 / 60 \mathrm{mmHg}$; pulse rate $140 \mathrm{bpm}$; respiratory rate $58 \mathrm{bpm}$; and oral temperature $38.5^{\circ} \mathrm{C}$. Laboratory assays were conducted to evaluate overall health and detect a disorder. A summary of the clinical outcomes is given in Table 1 . The serum levels of alanine transferase (ALT), aspartate amino transferase (AST), and alkaline phosphatase were in the normal range. Total protein and CRP-1h levels were in the normal range. Echocardiography (ECG) did not reveal any abnormalities (mild TR, good LV function, normal PAP). Following more evaluations, we found evidence of crackle auscultation, and decreased auscultation in the patient.

On the day of hospitalization, the patient presented with acute respiratory distress, tachycardia, and tachypnea without any cyanosis. Concerning the positive result of laboratory-confirmed COVID-19 pneumonia (based on conventional PCR assay and sequencing of PCR amplicons using a throat swab) and fever, for the accurate confirmation of COVID-19 infection, we performed a chest CT scan. The chest CT scan revealed bilateral lung involvement with pleural effusion. The reduction in saturation (up to 67\%) caused the patient's condition to worsen on the day of COVID-19 pneumonia diagnosis, and the patient was hospitalized in the intensive care unit (ICU) and connected to a high-flow ventilator. In the ICU, the medication started with vancomycin IV $240 \mathrm{mg}$ (up to three doses), tamiflu 75 $\mathrm{mg}$ (three tablets), and azithromycin $60 \mathrm{mg}$. The chronology of events by the parents confirmed that their child had no close contact with a suspected or confirmed COVID-19 case in the close family. On the day of hospitalization, the patient's condition worsened, and the patient's PT, PTT, and D-dimer increased (Table 1). Eventually, all therapeutic care was unsuccessful, and the patient expired due to acute respiratory distress syndrome (ARDS).

On 24 March 2020, a 16-month-old male was referred to the Emergency Department of Shahid Beheshti hospital, Qom city, complaining of laryngomalacia, microcephaly, cerebral palsy (CP), and breathing distress. His medical history indicated tracheotomy and hospitalization history, and he received levebel and phenobarbital.

Upon admission to the hospital, he looked ill, and his vital signs were as follows: Blood pressure $85 / 50 \mathrm{mmHg}$; pulse rate $140 \mathrm{bpm}$; respiratory rate $35 \mathrm{bpm}$; and oral temperature $37.2^{\circ} \mathrm{C}$. To accurately evaluate the patient, laboratory tests were performed. The results are summarized in Table 1 . The serum levels of ALT, AST, and alkaline phosphatase were in the normal range. Echocardiography (ECG) did not indicate any abnormalities. Laboratoryconfirmed COVID-19 pneumonia was assessed using SARSCoV-2 conventional PCR assay and sequencing of PCR amplicons, which was reported to be positive. After performing a chest CT scan, we noticed bilateral lung involvement with pleural effusion. Due to the persistence of dyspnea and reduction of saturation (up to 58\%), the patient was admitted to the ICU and connected to a high-flow ventilator. Medication with vancomycin BD120 mg (up to three doses) and Kaletra PG $7.5 \mathrm{mg}$ was started following the first symptoms of SARS-CoV-2 infection to reduce pneumonia and acute respiratory distress. The infant's condition worsened on the second day of hospitalization, and the patient's PT, PTT, and INR increased (Table 1). Eventually, on 4 April 2020, the patient was expired due to ARDS.

\section{Discussion}

In this study, we documented two confirmed COVID19 pediatrics with presentations of severe symptoms leading to death. Among COVID-19 pediatrics, who were referred to Fatemeh Masoumeh Hospital in Qom city, a few people were hospitalized. In comparison with adults, the majority of the neonates were discharged from the clinic without any need for hospitalization or medication. However, some patients in our pediatrics clinics, particularly those with high-risk factors, presented severe symptoms that needed high-flow ventilation or admission to the ICU. These findings are in line with the findings from other countries.

The low number of patients is a great limitation to this study. Although, according to the findings, we conclude that in both confirmed COVID-19 pediatrics and adults, the past history of illness has a great role in the incidence of severe symptoms and even death. The other finding of the study is that COVID-19 may cause death in pediatrics, like in adults, in the infected patients without any background disease ( $n=1,50 \%$ ). Like adults, social distancing and suitable protective equipment are necessary for the protection of pediatrics from this lethal disease. Also, this matter is important to be considered by close family members who have been confirmed to have COVID-19, especially infected parents who have to be in close contact with their children. 


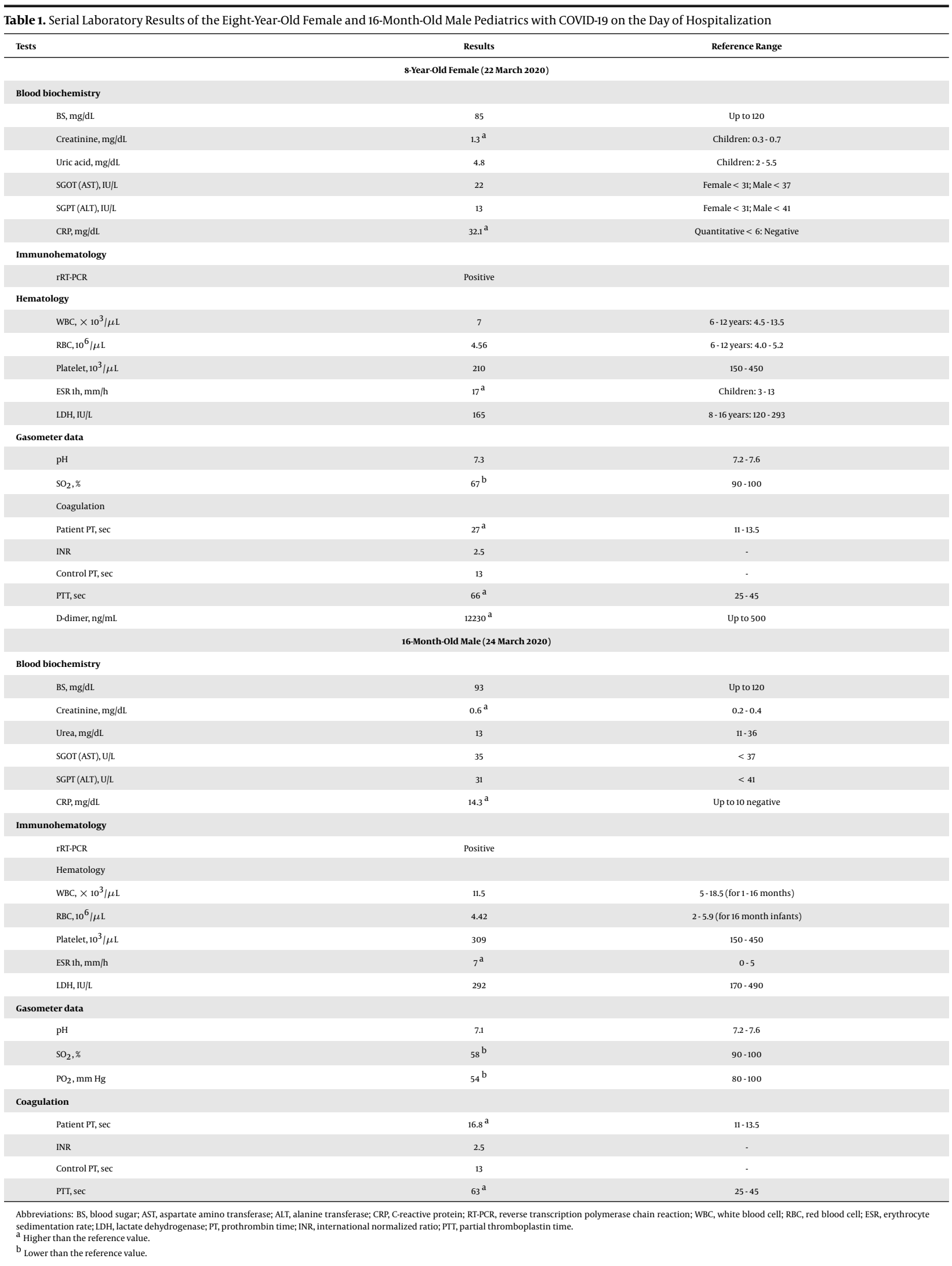




\section{Footnotes}

Authors' Contribution: Seyed Kamal Eshagh Hosaini writing of manuscript; Hosein Heydari, Ahmad Hormati and Mohammad Reza Ghadir, data analyzing and edit of manuscrict; Mahboubeh Afifian, collecting of data; Sajjad Ahmadpour, writing of manuscript and is corresponding author.

Conflict of Interests: The author declares no conflict of interest.

Ethical Approval: Approval was obtained from the Ethics Committee of Qom University of Medical Sciences. The procedures used in this study adhere to the tenets of the Declaration of Helsinki (No. IR.MUQ.REC.1399.056).

Funding/Support: There was no funding for this study.

\section{References}

1. Zhu N, Zhang D, Wang W, Li X, Yang B, Song J, et al. A novel Coronavirus from patients with Pneumonia in China, 2019. $N$ Engl J Med.
2020;382(8):727-33. doi: 10.1056/NEJMoa2001017. [PubMed: 31978945] [PubMed Central: PMC7092803].

2. Seyedi SJ, Shojaeian R, Hiradfar M, Mohammadipour A, Alamdaran SA Coronavirus Disease 2019 (COVID-19) outbreak in pediatrics and the role of pediatricians: A systematic review. Iran J Pediatr. 2020;30(2). e102784. doi: 10.5812/ijp.102784.

3. Ekrami Noghabi M, Baniasad A, Heidari E, Davoudian N, Malekzadeh F. A35-day old infant with COVID-19.Iran JPediatr. 2020;30(4). e103807. doi: 10.5812/ijp.103807.

4. Lu X, Zhang L, Du H, Zhang J, Li YY, Qu J, et al. SARS-CoV-2 infection in children. N Engl J Med. 2020;382(17):1663-5. doi: 10.1056/NEJMc2005073. [PubMed: 32187458]. [PubMed Central: PMC7121177].

5. Dong Y, Mo X, Hu Y, Qi X, Jiang F, Jiang Z, et al. Epidemiological characteristics of 2143 pediatric patients with 2019 coronavirus disease in China. Pediatrics. 2020;145(6). e20200702.

6. Hoehl S, Rabenau H, Berger A, Kortenbusch M, Cinatl J, Bojkova D, et al. Evidence of SARS-CoV-2 infection in returning travelers from Wuhan, China. NEngl J Med. 2020;382(13):1278-80. doi:10.1056/NEJMc2001899. [PubMed: 32069388]. [PubMed Central: PMC7121749].

7. Hormati A, Ghadir MR, Zamani F, Khodadadi J, Afifian M, Ahmadpour S. Preventive strategies used by GI physicians during the COVID-19 pandemic. New Microbes New Infect. 2020;35:100676. doi 10.1016/j.nmni.2020.100676. [PubMed: 32292589]. [PubMed Central: PMC7141622] 